\title{
Recombination Reactions of Atomic Chlorine in Compressed Gases. 3. Molecular Dynamics and Smoluchowski Equation Studies with Argon Pressure up to 6 kbar
}

\author{
T.-T. Song, Y.-S. Hwang, and T.-M. Su* \\ Department of Chemistry, National Taiwan University, Taipei, Taiwan, R. O. C., and The Institute of the \\ Atomic and Molecular Sciences, Academia Sinica, Taipei, Taiwan, R. O. C.
}

Received: July 31, 1996; In Final Form: December 12, $1996^{\otimes}$

\begin{abstract}
The photodissociation/recombination reactions of chlorine molecules under $355 \mathrm{~nm}$ laser radiation in compressed argon gas were studied by canonical molecular dynamics with the Nose-Hoover thermostat. The thermal relaxation and the final thermalized spatial chlorine atom-pair distribution over the dissociating electronic potential were analyzed and also compared with the predictions of the Langevin equation approach. The geminate recombination probability rate constants and quantum yields for all the bound electronic states of the chlorine molecule were calculated. The recombination in the ground electronic state was also studied by the Smoluchowski equation approach. Comparisons between the results of the molecular dynamics and the Smoluchowski equation approaches suggest that the solutions of the simplest diffusion equation consistently underestimate both the geminate rate constants and the quantum yields. Factors that may affect the recombination rate constants and quantum yields in the Smoluchowski equation approach were evaluated. They include local temperature jump due to initial hot atom thermalization, non-Markovian effect, meanforce potential, $\mathrm{Cl}-\mathrm{Cl}$ long-range potential, and hydrodynamic interactions. For the $\mathrm{Cl}_{2} / \mathrm{Ar}$ system, it was found that a near quantitative agreement could be obtained by just taking the non-Markovian effect into account in the framework of the generalized diffusion equation approach. The contribution of mean-force potential, $\mathrm{Cl}-\mathrm{Cl}$ long-range potential, and hydrodynamic interactions could account for the remaining mismatch in the short time and medium time domains. The effect of local temperature jump due to initial hot atom thermalization was found to be insignificant.
\end{abstract}

\section{Introduction}

The photodissociation/recombination reactions of the molecular halogens in compressed rare gases have received continual attention for more than two decades. ${ }^{1-13}$ They are generally regarded as model reactions for the study of the photolytic cage effect in the bulk systems. Experimentally, for the earlier studies, the photolysis quantum yields and the nongeminate atomic recombination rate constants of iodine, bromine, and chlorine were measured and the mechanisms for geminate recombination were proposed. ${ }^{1-6}$ Along this general line, the onset of the geminate recombination reactions of the chlorine molecules in the lower pressure region was investigated recently. ${ }^{12,13}$ Meanwhile, in another different experimental development, the geminate recombinations of the $\mathrm{I}_{2} / \mathrm{Xe}$ system were intensively studied by the picosecond transient absorption method. ${ }^{7,8}$ In the past few years, the real-time dynamics of the iodine dissociation/recombination reactions in the rare gas cages over a wide range of gas pressures have been investigated with femtosecond time resolution. ${ }^{9-11}$

Theoretically, the photodissociation/recombination reactions of iodine and bromine have been studied by the molecular dynamics (MD) simulations for some time. ${ }^{14-17}$ Most of the systems studied were in the form of either the low-temperature Ar clusters or solid Ar, which all correspond to the lower side of the system temperatures. The short time dynamics were usually emphasized. They served as a theoretical guidance for the microscopic understanding of the corresponding experimental observations, especially for those experiments with femtosecond time resolution. ${ }^{18,19}$

For the photodissociation/recombination processes of the halogen/rare gas systems under constant temperature, a condition

\footnotetext{
${ }^{\otimes}$ Abstract published in Advance ACS Abstracts, April 15, 1997.
}

under which most of the bulk experiments were carried out, there were no reported MD studies that directly addressed these more practical measurements. In the first part of the present work, Nose-Hoover molecular dynamics ${ }^{20-22}$ was employed to study the photodissociation/recombination of the $\mathrm{Cl}_{2} / \mathrm{Ar}$ system under $355 \mathrm{~nm}$ radiation at $300 \mathrm{~K}$. The hot atom thermalization process and the final thermalized chlorine atompair spatial distribution over the dissociating electronic potential were analyzed. The geminate recombination rate constants and quantum yields for all the bound electronic states of the chlorine molecule were calculated.

Besides the MD methods, one may take a classical phenomenological approach. Under high enough rare gas density, the photodissociation/recombination of the halogen/rare gas systems could be treated as diffusion-influenced reactions ${ }^{6,23}$ and solved in the framework of the diffusion equation according to the theory of Smoluchowski and that of Collins and Kimball. ${ }^{24,25}$ It has been shown that the measured density dependencies of the photolysis quantum yields of the halogen/rare gas systems are in reasonably good agreement with the theoretical predictions even though the phenomenological parameters were necessarily determined separately by either experiments or estimations in these studies. ${ }^{6,11,23}$ Along this line, recently, in order to probe the limit of the diffusion equation methods, especially in the short time region, comparisons have been made between the results of the MD simulations and the predictions of the Smoluchowski theory for diffusion-influenced reactions in a dense hard-sphere liquid. ${ }^{26-28}$

In the second part of the present work, the geminate recombination rate constants and photolysis quantum yields of $\mathrm{Cl}_{2} / \mathrm{Ar}$ in the ground electronic state were calculated according to the framework of the Smoluchowski equation. The results were compared with those of the corresponding MD simulations. 
The effect of local heating due to the transient energy dissipation of the hot chlorine atoms on the geminate recombination rates, a condition frequently encountered in experiments but only explicitly considered recently in the framework of the diffusion equation, ${ }^{29}$ was evaluated. The contributions of non-Markovian effect, mean-force potential, $\mathrm{Cl}-\mathrm{Cl}$ long-range potential, and hydrodynamic interactions were also calculated for the present system. ${ }^{26-28}$ The present molecular system is simple enough such that the experimental measurements, molecular dynamics simulations, and the predictions of the Smoluchowski theory based on the Smoluchowski equations could be carried out or calculated in a more definite manner. Eventually, the accuracy of these theoretical methodologies could be evaluated by checking against the corresponding experimental results of these simple systems.

\section{Systems and Methods of Molecular Dynamics Calculations}

2A. Potential Energy Functions for the $\mathbf{C l}_{2} / \mathbf{A r}$ System. The Ar-Ar interaction potential is represented by the LennardJones 6-12 potential with parameters $\epsilon_{\mathrm{Ar}-\mathrm{Ar}}=83.3 \mathrm{~cm}^{-1}$ and $\sigma_{\mathrm{Ar}-\mathrm{Ar}}=3.41 \AA .^{30}$ For the $\mathrm{Cl}-\mathrm{Ar}$ potential, it has been recognized that the interaction potentials of the halogen-atom/ rare-gas systems are close to those of the corresponding raregas/rare-gas systems as suggested in the molecular beam scattering experiments. ${ }^{31,32}$ The similarity of the transport properties between the $\mathrm{Cl} / \mathrm{Ar}$ and $\mathrm{Ar} / \mathrm{Ar}$ systems also supports that the $\mathrm{Cl}-\mathrm{Ar}$ potential could be well-approximated by the above $\mathrm{Ar}-\mathrm{Ar}$ potential. ${ }^{33}$ In this study, the $\mathrm{Ar}-\mathrm{Ar}$ potential form was adapted directly for the $\mathrm{Cl}-\mathrm{Ar}$ potential. The $\mathrm{Ar}-$ $\mathrm{Cl}_{2}$ potential was then calculated by the atom-atom additivity model. ${ }^{34}$ With these proposed interaction potentials and model, the equilibrium bond energy of $\mathrm{Ar}-\mathrm{Cl}_{2}$ is $167 \mathrm{~cm}^{-1}$, and the equilibrium bond distance from $\mathrm{Ar}$ to the center of mass of $\mathrm{Cl}_{2}$ is $3.69 \AA$. These values are in good agreement with the experimental bond energies of $188 \mathrm{~cm}^{-1}$ for the $\mathrm{X}$ state, 178 $\mathrm{cm}^{-1}$ for the $\mathrm{B}$ state, and the experimental bond distance of 3.7 $\AA$ for both states of the $\mathrm{Ar}-\mathrm{Cl}_{2}$ complex. ${ }^{34}$

Before the photodissociation, the ground state ${ }^{1} \sum_{\mathrm{g}}{ }^{+}(\mathrm{X})$ of $\mathrm{Cl}_{2}$ is represented by the Morse function with parameters determined by the spectroscopic data: $\omega_{\mathrm{e}}=559.7 \mathrm{~cm}^{-1}, \omega_{\mathrm{e}} \chi_{\mathrm{e}}$ $=2.675 \mathrm{~cm}^{-1}$, and $\mathrm{r}_{\mathrm{e}}=1.988 \AA .{ }^{35}$ This potential was employed to establish the initial thermal equilibrium of the chlorine molecule in the MD systems. The potential energy function of ${ }^{1} \Pi_{1 \mathrm{u}}$ is expressed as ${ }^{36}$

$$
\begin{array}{rlrl}
U\left({ }^{1} \Pi_{1 \mathrm{u}}\right) & =U_{0}-D_{\mathrm{e}}+2.530 \times 10^{4} / \rho^{2} & & r<2.152 \AA \\
& =1.243 \times 10^{6} / r^{8} & r \geq 2.152 \AA
\end{array}
$$

in which $U_{0}=3617 \mathrm{~cm}^{-1}, D_{\mathrm{e}}=2.028 \times 10^{4} \mathrm{~cm}^{-1}$, and $\rho=$ $(r-1.009) \AA$. The second switching potential of eq 1 was chosen just for a smoother repulsive decay as the internuclear distance is longer than $2.152 \AA$. The exact form for this part of the potential is not available. Nevertheless, the results of the present MD simulations are not sensitive to the functional forms of this repulsive potential tail.

In the recombination process, the two ground state chlorine atoms may recombine to four possible bound states: $\mathrm{X}^{1} \Sigma_{\mathrm{g}}{ }^{+}, 37$ $\mathrm{A}^{3} \Pi\left(1_{\mathrm{u}}\right),{ }^{38} \mathrm{~A}^{\prime}{ }^{3} \Pi\left(2_{\mathrm{u}}\right),{ }^{39}$ and $\mathrm{B}^{\prime}{ }^{3} \Pi_{\mathrm{u}}\left(0_{\mathrm{u}}^{-}\right) .{ }^{40}$ From their Rydberg-Klein-Rees potential points, these potentials were represented by the following analytic functions, which emphasize the fitting accuracy of the attractive potential branch, especially the medium- to long-range part of the potentials to within 40

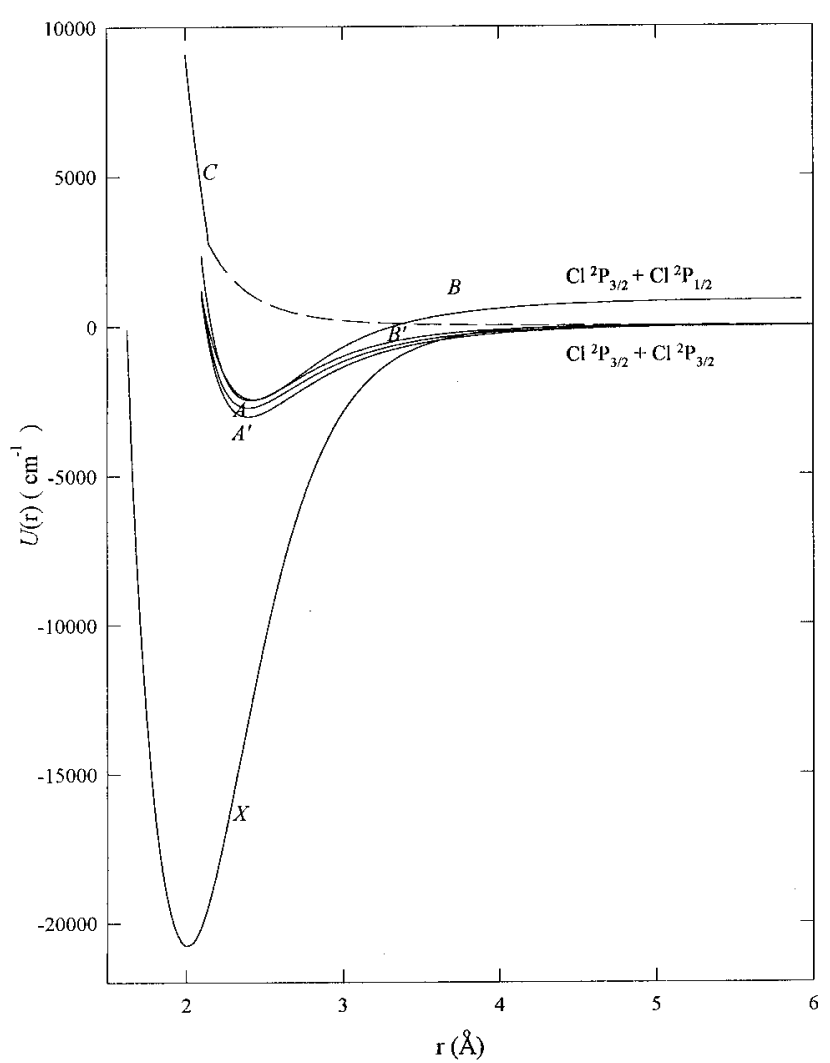

Figure 1. Potential energy curves of the $\mathrm{X}, \mathrm{A}^{\prime}, \mathrm{A}, \mathrm{B}^{\prime}, \mathrm{B}$, and $\mathrm{C}$ states of $\mathrm{Cl}_{2}$ as calculated by the analytic potential functions for the MD calculations. See text for details.

TABLE 1: Fitted Parameters of the Potential Functions for the States $A, A^{\prime}$, and $B^{\prime}$

\begin{tabular}{lrrrc}
\hline & \multicolumn{1}{c}{$\mathrm{A}$} & \multicolumn{1}{c}{$\mathrm{A}^{\prime}$} & \multicolumn{1}{c}{$\mathrm{B}^{\prime}$} & units \\
\hline$C_{6}$ & $4.268 \times 10^{5}$ & $7.912 \times 10^{5}$ & $-2.308 \times 10^{5}$ & $\mathrm{~cm}^{-1} \AA^{6}$ \\
$C_{8}$ & $1.003 \times 10^{7}$ & $7.486 \times 10^{6}$ & $1.694 \times 10^{7}$ & $\mathrm{~cm}^{-1} \AA^{8}$ \\
$C_{10}$ & $-5.456 \times 10^{7}$ & $-5.010 \times 10^{7}$ & $-7.416 \times 10^{7}$ & $\mathrm{~cm}^{-1} \AA^{10}$
\end{tabular}

$\mathrm{cm}^{-1}$. The $\mathrm{X}$ state potential is represented by

$$
\begin{aligned}
U(\mathrm{X}) & =2.5937 \times 10^{4} \times \\
& \left\{\left[1-\mathrm{e}^{-2.9685(r-1.588)}\right]^{8}-1\right\}+2.6669 \times 10^{6} / r^{9.539}
\end{aligned}
$$

in which the energy zero is set at the dissociation limit of the chlorine molecule, the energy is in $\mathrm{cm}^{-1}$, and the distance is in angstroms. The remaining three states are represented by the functional form

$$
U\left(\mathrm{~A}, \mathrm{~A}^{\prime}, \text { or } \mathrm{B}^{\prime}\right)=-\sum_{n} C_{n} / r^{n}
$$

where $C_{n}$ 's are fitted parameters with $n=6,8$, and 10. Table 1 lists the fitted values, and Figure 1 shows the electronic potentials of the chlorine molecule needed in the present study.

2B. Calculation Methods and Procedures. Since most of the photodissociation and recombination experiments were carried out at constant temperature instead of constant energy condition and also the recombination rate constants are sensitive to the temperature, to simulate these experiments, the NVT ensemble is a more direct choice for the calculations. In this study the Nose-Hoover molecular dynamics, which gives a canonical ensemble, was employed. ${ }^{20-22,30}$

The simulated particle number was either 500 or 108 , of which two are the chlorine atoms. At $300 \mathrm{~K}$, for a given experimental argon pressure, the simulation volume size was determined from the corresponding experimental argon density 
TABLE 2: Fitted Parameters of the Atom-Pair Spatial Distributions, Thermalization Times, Thermalization Rate Constants, Friction Constants, and Diffusion Constants of Chlorine Atoms in Ar at $300 \mathrm{~K}$

\begin{tabular}{cccccccccc}
\hline pressure $(\mathrm{kbar})$ & $b / 10^{-2}(1 / \AA)^{2}$ & $c(\mathrm{rad})^{-1}$ & $r_{0(\max )}(\AA)$ & $\Delta r^{a}(\AA)$ & $\vartheta_{0(\max )}(\mathrm{deg})$ & $t_{\text {therm }}^{b}(\mathrm{ps})$ & $k_{\text {therm }^{c}}(\mathrm{ps})^{-1}$ & $\xi^{d}(\mathrm{ps})^{-1}$ & $D_{0(\mathrm{Cl}-\mathrm{Ar})}{ }^{e}\left(10^{-8} \mathrm{~m}^{2} \mathrm{~s}^{-1}\right)$ \\
\hline 0.18 & 0.081 & 5.04 & 36.9 & 45.3 & 11.2 & 3.74 & 0.81 & 0.77 & 9.18 \\
0.50 & 0.823 & 5.87 & 13.0 & 18.0 & 9.66 & 1.25 & 2.21 & 2.07 & 3.39 \\
1.00 & 2.51 & 7.40 & 8.35 & 11.1 & 7.70 & 0.683 & 4.90 & 3.65 & 1.93 \\
2.00 & 6.12 & 9.04 & 6.21 & 7.96 & 6.32 & 0.460 & 7.39 & 5.56 & 1.27 \\
4.00 & 14.6 & 10.5 & 4.96 & 5.49 & 5.46 & 0.283 & 10.7 & 9.29 & 0.742 \\
6.00 & 23.9 & 13.3 & 4.51 & 4.31 & 4.30 & 0.314 & 12.3 & 14.3 & 0.492
\end{tabular}

${ }^{a}$ Chlorine atom-pair separations calculated by the Langevin equation. ${ }^{b}$ Average thermalization times for hot atoms to reach a kinetic energy of $2 k T$ calculated by the MD method. ${ }^{c}$ Thermalization rate constant fitted by the exponential functional form. ${ }^{d} \xi m$ is the friction constants of the chlorine atom in Ar; $m$ is the chlorine atom mass. ${ }^{e}$ Diffusion constants of chlorine atoms in Ar calculated by the MD method.

available in the literature. ${ }^{41,42}$ To check the internal consistency among the interaction potentials, the MD method, and the thermodynamic properties, the theoretical argon pressures of the net argon system were calculated with the above $\mathrm{Ar}-\mathrm{Ar}$ interaction potential. Over the pressure range $0.50-6.00 \mathrm{kbar}$, the average percentage deviation between the calculated and experimental argon pressures is $1.8 \%$. The calculated selfdiffusion constants of the argon atoms are also in good agreement with the few experimental values in the literature. ${ }^{43,44}$ The diffusion constants of chlorine atoms in Ar over 0.18$6.00 \mathrm{kbar}$ at $300 \mathrm{~K}$ were then calculated by the MD method and are listed in the last column of Table 2.

The experiments of the photodissociation/recombination of the chlorine molecules by $355 \mathrm{~nm}$ photons under argon buffer pressure from 0.50 to $6.00 \mathrm{kbar}$ were simulated. The systems were set at $300 \mathrm{~K}$. For the simulation of the initial hot atom thermalization and their spatial distributions, 500 particles of which two are the chlorine atom-pair, were used. For the simulation of the complete photodissociation and recombination process, 108 particles, of which two are the chlorine atom-pair, were employed. The periodic boundary condition was imposed and the pair potential was truncated at $8.5 \AA\left(2.5 \sigma_{\mathrm{Ar}-\mathrm{Ar}}\right)$ except for the interaction between the chlorine atoms. ${ }^{30}$ The real internuclear distance and the potential energy of the geminate chlorine atoms were always tracked and calculated even if the chlorine atom image is in the central cell, as dictated by the periodic boundary condition. Except for the lower pressure cases, in which the integration step was 2 fs and the time span was $400 \mathrm{ps}$, the remaining simulations used an integration step of $1 \mathrm{fs}$ and a total of $100 \mathrm{ps}$ after the photodissociation. The structural and the related dynamic information was sampled and recorded. Each simulation was always preceded by a 5 ps predissociation period for the thermal equilibration.

At the moment of photodissociation, the ground state chlorine molecule was switched to the ${ }^{1} \Pi_{1 \mathrm{u}}$ exited state, which is a pure repulsive state. The hot atom thermalization and the atom-pair spatial distributions were obtained at this potential. For the recombination reaction, the potential surface was switched to the desired bound state potential as the chlorine atom-pair reaches the point where the potential energy separation is less then $(1 / 2) k T$. The trajectories of the chlorine atom-pair then follow the new designated potential all the way to the preset time of the simulation, which is either $100 \mathrm{ps}$ for the highpressure cases or 400 ps for the low-pressure ones.

\section{Smoluchowski Equation Approach}

As suggested in the above MD calculations, the hot chlorine atom-pairs generated by photodissociation reach their thermal equilibrium state in specific spatial distributions. In this section, we shall treat the recombination reactions of these geminate atom-pairs as the two-body diffusion problem. The corresponding Smoluchowski equation was then solved with the above initial spatial distributions under specific boundary conditions.
The solutions of the field-free two-body diffusion problem and its following variations considered in this report are expressed in closed form. The Smoluchowski equation with either the mean-force potential or also including the hydrodynamic interactions shall be solved by Brownian dynamics trajectory simulations.

3A. The Solutions of the Field-Free Two-Body Diffusion Problem. As shown by Scheider, ${ }^{45}$ the conditional probability for the field-free two-body recombination reaction that obeys the diffusion equation with radiation boundary condition can be written as

$$
\begin{aligned}
\Gamma\left(\mathbf{r}, t \mid \mathbf{r}_{0}, d, h\right)=\frac{1}{2 \pi^{2} d^{2} l=0} \sum_{l=0}^{\infty}(2 l & +1) P_{l} \times \\
& (\cos \gamma) \int_{0}^{\infty} \mathrm{d} k \frac{y_{l}(r) y_{l}\left(r_{0}\right)}{N_{l}^{2}} \mathrm{e}^{-D_{0} k^{2} t}
\end{aligned}
$$

with

$$
\begin{aligned}
& y_{l}(r)=(k d)^{2}\left\{\left[j_{l}(k r) y_{l}^{\prime}(k d)-y_{l}(k r) j_{l}^{\prime}(k d)\right]-\right. \\
& \left.(h / k)\left[j_{l}(k r) y_{l}(k d)-y_{l}(k r) j_{l}(k d)\right]\right\}
\end{aligned}
$$

and

$$
N_{l}^{2}=(k d)^{2}\left\{\left[\frac{h}{k} j_{l}(k d)-j_{l}^{\prime}(k d)\right]^{2}-\left[\frac{h}{k} y_{l}(k d)-y_{l}^{\prime}(k d)\right]^{2}\right\}
$$

where $\Gamma$ is the probability density of finding $B$ at the spacetime of $(\mathbf{r}, t)$ if initially it was at $\left(\mathbf{r}_{0}, 0\right), D_{0}$ is the sum of the individual diffusion coefficients of the reacting pair $\mathrm{A}$ and $\mathrm{B}$, $\gamma$ is the angle between the vectors $\mathbf{r}$ and $\mathbf{r}_{\mathbf{0}}, j_{l}$ and $y_{l}$ are the first and second spherical Bessel functions, respectively, and $d$ and $h$ are defined by the radiation boundary condition

$$
\left.\frac{\partial \Gamma\left(\mathbf{r}, t \mid \mathbf{r}_{0}\right)}{\partial r}\right|_{r=d}=h \Gamma\left(\mathbf{d}, t \mid \mathbf{r}_{0}\right)
$$

The initial condition is $\lim _{t \rightarrow 0} \Gamma\left(\mathbf{r}, t \mid \mathbf{r}_{\mathbf{0}}\right)=\delta\left(\mathbf{r}_{\mathbf{0}}-\mathbf{r}\right)$.

The probability rate of reaction over the entire reaction surface $\Sigma$ due to an instantaneous point source at $\left(\mathbf{r}_{\mathbf{0}}, t=0\right)$ can be calculated by

$$
R\left(\mathbf{r}_{0}, t\right)=\int_{\Sigma} D_{0} h \Gamma\left(\mathbf{d}, t \mid \mathbf{r}_{0}, d, h\right) \mathrm{d} \sigma
$$

$$
\begin{array}{r}
r_{0} \sqrt{\frac{D_{0}}{\pi t}} \mathrm{e}^{-\left(r_{0}-d\right)^{2} / 4 D_{0} t}-\frac{\bar{h} D_{0} d h}{r_{0}} \mathrm{e}^{D_{0} \bar{h}^{2} t+\left(r_{0}-d\right) \bar{h}} \operatorname{erfc}\left(\bar{h} \sqrt{D_{0} t}+\right. \\
\left.\frac{r_{0}-d}{\sqrt{4 D_{0} t}}\right)
\end{array}
$$

with 


$$
\bar{h}=h+1 / d
$$

where $\mathrm{d} \sigma=d^{2} \sin \theta \mathrm{d} \theta \mathrm{d} \phi$. For a nonuniform initial spatial distribution, $f\left(\mathbf{r}_{0}\right)$, the total probability rate of reaction becomes

$$
R(t)=\iiint R\left(\mathbf{r}_{0}, t\right) f\left(\mathbf{r}_{0}\right) \mathrm{d}^{3} \mathbf{r}_{0}
$$

with

$$
\begin{aligned}
f\left(\mathbf{r}_{0}\right) & =N\left(r_{0}-R_{0}\right)^{2} \exp \left(-b r_{0}{ }^{2}-c \theta_{0}\right) \sin \theta_{0} / r_{0}{ }^{2} & & \left(r_{0} \geq R_{0}\right) \\
& =0 & & \left(r_{0}<R_{0}\right)
\end{aligned}
$$

in which $N$ is the normalization constant, and $R_{0}, b$, and $c$ are parameters to be determined by the MD calculations. The details of the distribution function shall be discussed in the next section.

After integration, the probability reaction rate becomes

$$
\begin{aligned}
& R(t)= \frac{4 \pi N d h}{c\left(c^{2}+4\right)}\left(1-\mathrm{e}^{-c \pi}\right) \times \\
& {\left[\sqrt{\frac{D_{0}}{\pi t}} \int_{R_{0}}^{\infty} \frac{\left(r_{0}-R_{0}\right)^{2}}{r_{0}} \mathrm{e}^{-\left[\left(r_{0}-d\right)^{2}+4 b r_{0}{ }^{2} D_{0} t\right] / 4 D_{0} t} \mathrm{~d} r_{0}-\right.} \\
& \bar{h} D_{0} \mathrm{e}^{\left(\bar{h}^{2} D_{0} t-d \bar{h}\right)} \int_{R_{0} \frac{\left(r_{0}-R_{0}\right)^{2}}{r_{0}} \mathrm{e}^{-\left(b r_{0}{ }^{2}-r_{0} \bar{h}\right)}}^{\operatorname{erfc}\left(\bar{h} \sqrt{D_{0} t}+\right.} \\
&\left.\left.\frac{r_{0}-d}{\sqrt{4 D_{0} t}}\right) \mathrm{~d} r_{0}\right]
\end{aligned}
$$

Under the Smoluchowski boundary condition, which is equivalent to the condition of $h \rightarrow \infty$, eq 12 becomes

$$
\begin{aligned}
& R_{\mathrm{S}}(t)=\frac{2 N d \pi^{1 / 2} D_{0}^{-1 / 2} t^{-3 / 2}}{c\left(c^{2}+4\right)}(1- \\
&\left.\mathrm{e}^{-c \pi}\right) \int_{R_{0}}^{\infty} \frac{\left(r_{0}-R_{0}\right)^{2}\left(r_{0}-d\right)}{r_{0}} \mathrm{e}^{\left.-\left[\left(r_{0}-d\right)^{2}+4 b r_{0}{ }^{2} D_{0}\right]\right] / 4 D_{0} t} \mathrm{~d} r_{0}
\end{aligned}
$$

The cumulative reaction probability due to an instantaneous point source at $\left(\mathbf{r}_{\mathbf{0}}, t=0\right)$ is defined as

$$
\begin{array}{r}
W\left(\mathbf{r}_{0}, t\right)=\int_{0}^{t} R\left(\mathbf{r}_{0}, t^{\prime}\right) \mathrm{d} t^{\prime} \\
=\frac{d h}{\bar{h} r_{0}}\left[\operatorname{erfc}\left(\frac{r_{0}-d}{\sqrt{4 D_{0} t}}\right)-\mathrm{e}^{\bar{h}^{2} D_{0} t+\left(r_{0}-d\right) \bar{h}} \operatorname{erfc}\left(\bar{h} \sqrt{D_{0} t}+\right.\right. \\
\left.\left.\frac{r_{0}-d}{\sqrt{4 D_{0}} t}\right)\right]
\end{array}
$$

Integrating over the initial spatial distribution, one obtains the cumulative reaction probability

$$
\begin{aligned}
& W(t)=\frac{4 \pi N d h\left(1-\mathrm{e}^{-c \pi}\right)}{\bar{h} c\left(c^{2}+4\right)} \times \int_{R_{0}}^{\infty} \frac{\left(r_{0}-R_{0}\right)^{2}}{r_{0}} \mathrm{e}^{-b r_{0}{ }^{2}} \times \\
& {\left[\operatorname{erfc}\left(\frac{r_{0}-d}{\sqrt{4 D_{0} t}}\right)-\mathrm{e}^{\bar{h}^{2} D_{0} t+\left(r_{0}-d\right) \bar{h}} \operatorname{erfc}\left(\bar{h} \sqrt{D_{0} t}+\frac{r_{0}-d}{\sqrt{4 D_{0} t}}\right)\right] \mathrm{d} r_{0}}
\end{aligned}
$$

Under the Smoluchowski boundary, eq 15 becomes

$$
\begin{array}{r}
W_{\mathrm{S}}(t)=\frac{4 \pi N d\left(1-\mathrm{e}^{-c \pi}\right)}{c\left(c^{2}+4\right)} \int_{R_{0}}^{\infty} \frac{\left(r_{0}-R_{0}\right)^{2}}{r_{0}} \mathrm{e}^{-b r_{0}^{2}} \times \\
\operatorname{erfc}\left(\frac{r_{0}-d}{\sqrt{4 D_{0} t}}\right) \mathrm{d} r_{0}
\end{array}
$$

3B. The Solutions of the Smoluchowski Equation Calculated by Brownian Dynamics Trajectory Simulations. For the calculations of the contribution of mean-force potential, $\mathrm{Cl}-$ $\mathrm{Cl}$ long-range potential, and hydrodynamic interactions in the present system, the Smoluchowski equations with general interaction potentials and/or spatially dependent diffusion coefficients were employed. ${ }^{46-48}$ The solutions of the timedependent probability reaction rate and cumulative reaction probability have to be calculated numerically. For the present purpose, the Brownian dynamics trajectory simulations, which were developed to handle arbitrary potentials and distancedependent diffusion coefficients, were employed to calculate these time-dependent quantities. ${ }^{27,49,50}$

\section{Results of the Molecular Dynamics Method and the Smoluchowski Equation Approach}

4A. The Hot Chlorine Atom Thermalization and Geminate Chlorine Atom-Pair Spatial Distributions. After the absorption of a $355 \mathrm{~nm}$ photon, in the initial stage, the chlorine atom-pair follows the $\mathrm{C}^{1} \Pi_{1 \mathrm{u}}$ repulsive electronic state and undergo hot atom thermalization process in the Ar buffer gas. Figure 2 shows the trajectory-averaged speed relaxation of the chlorine atom at four different argon buffer pressures. Note that before the photoexcitation the speed of the center-of-mass of the chlorine molecules was registered. As shown in the figure, after the excitation, depending on the buffer pressures, the dissociating atoms always take some finite time to reach their maximum speeds. The high-frequency modulation is due to the coupling of the motion of the chlorine atom to the NoseHoover thermostat. ${ }^{20-22}$ The mean thermalization times of the hot atoms under various argon pressures, which is defined as the average time for the hot atom to reach a kinetic energy of $2 k T$-an energy with $1 / 2 k T$ above the thermal energy-were determined accordingly. As shown in Table 1, the mean thermalization time decreases from 3.74 to 0.283 ps as the argon pressure increases from 0.180 to $4.00 \mathrm{kbar}$. However, it slightly increases to $0.314 \mathrm{ps}$ at $6.00 \mathrm{kbar}$ argon pressure. The reverse trend in the high-pressure region is just the consequence of the backscattering of the hot chlorine atoms by the neighboring argon atoms which form a more effective cage around the chlorine molecules as the argon pressure is high enough. The similarity of the atomic masses between the chlorine and argon atoms also enhances this effect.

As shown by the dashed curves in Figure 2, the relaxation speed could be fitted by a simple exponential decay function over the present pressure range. The fitted decay rate constants $k_{\text {therm }}$ are listed in Table 2. Phenomenologically, one may also evaluate the relaxation speed rate constants by the Langevin equation. ${ }^{48}$ Neglecting the contribution of thermal energy, the root mean square speed of the hot chlorine atoms decays exponentially with a rate constant of $\zeta=\gamma^{\prime} / m$, in which $\gamma^{\prime}$ is the friction constant and $m$ is the chlorine atom mass. The $\zeta$ values, which are calculated from the corresponding diffusion constants with the Einstein relation, are also listed in Table 2. The good agreement between the values of $k_{\text {therm }}$ and $\zeta$ over the present pressure range suggests that the thermal relaxation process in the $\mathrm{Cl} / \mathrm{Ar}$ system could be described by the Langevin equation reasonably well. 


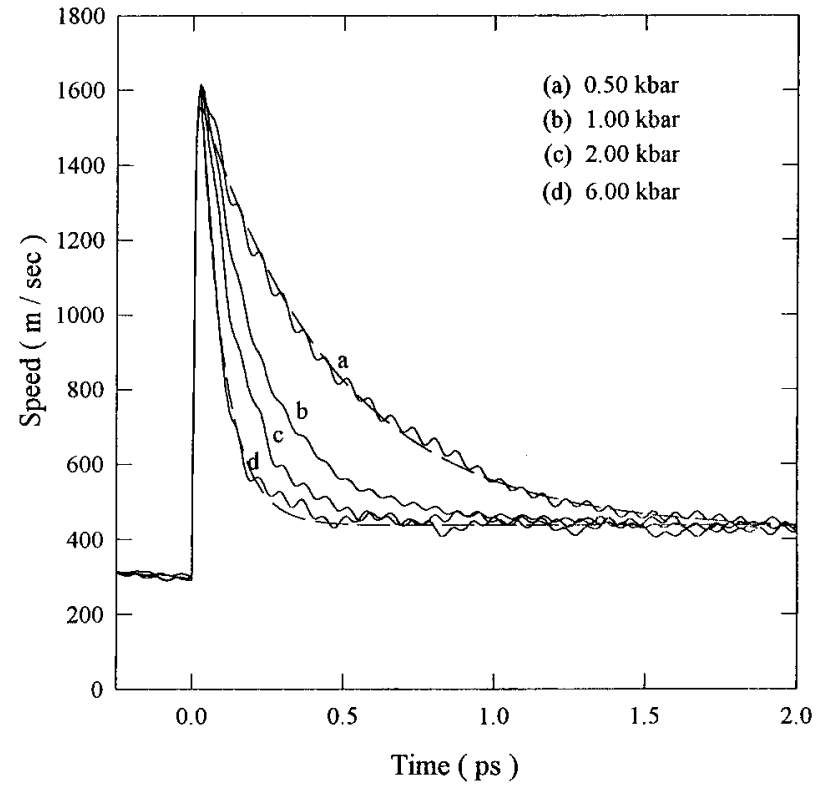

Figure 2. Relaxation speed of the chlorine atom after $355 \mathrm{~nm}$ photon absorption. Before the excitation, the speed of the center-of-mass of the chlorine molecule was recorded. The high-frequency modulation of the relaxation is due to the coupling between the system and the thermostat. The relaxation could be well-represented by a simple exponential decay function, as shown by the dashed fitted lines.

The mean thermalization times were then used as the stop time for the determination of the relative spatial distributions of the chlorine atom-pairs. The internuclear coordinates of the chlorine atom-pair at $t=0$, i.e. the initial molecular chlorine, are set as the reference coordinates. Depending on the argon pressure, over 1000 trajectories were usually taken. The normalized spatial probability distributions were then fitted with the functional form of eq 11. In considering the proposed functional form, the following three factors were taken into account. (a) The first factor is the physical size of the chlorine atoms. The repulsive nature of the electronic potential and the extra initial translational energy prevent the atom-pair from coming closer to each other to within a certain distance. This distance was designated as $R_{0}$ and is weakly dependent on both the buffer gas density and the initial atom speed. For the present purpose, it was set at $3.58 \AA$, an adequate value which could take account of both the spatial distribution of the MD calculations and also the reaction boundary radius in the diffusion reaction theory. (b) The second factor is the radial probability distribution. If neglecting the particle size, it is assumed to be in the Gaussian form as suggested in the general functional form of the probability distribution derived from the Langevin equation. ${ }^{51}$ (c) The third factor is the angular probability distribution. The azimuthal angle of the maximum probability distribution, which is shifted from $0^{\circ}$ due to the rotational motion of the parent molecule, should be properly represented and its low-pressure asymptotic value should approach that of the gas-phase photofragment spectroscopic measurements in the molecular beam experiments. ${ }^{52}$

Equation 11 is the simplest form that meets the above requirements and also is a good representation of the present MD data. Figure 3 shows a typical probability density contour map of eq 11 at $2.00 \mathrm{kbar}$ argon pressure. Following the equation, the internuclear distance of the maximum radial probability density per unit radial distance is

$$
r_{0(\max )}={ }_{1}^{1}{ }_{2}\left(R_{0}+\sqrt{R_{0}^{2}+4 / b}\right)
$$

and the azimuthal angle of the maximum angular probability

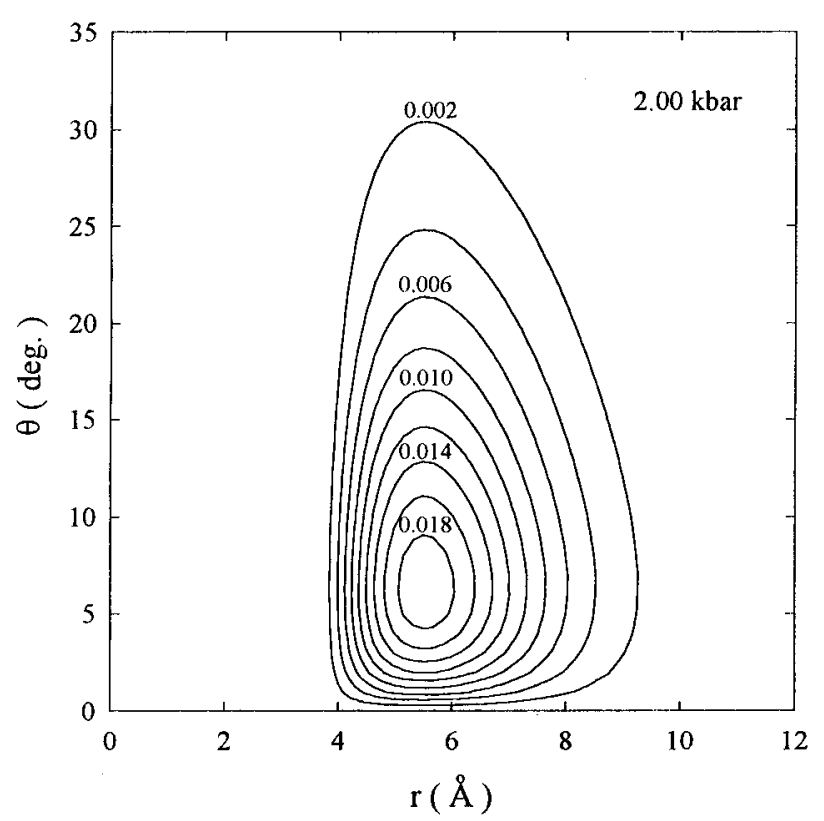

Figure 3. Contour map of the spatial probability distribution of the dissociated chlorine atom-pairs at $2.00 \mathrm{kbar}$ argon, the functional form of eq 11 .

density per unit solid angle is

$$
\vartheta_{0(\max )}=\tan ^{-1}(1 / c)
$$

This angle is due to the thermal rotational motion of the parent chlorine molecules. Table 2 lists the values of the fitted parameters and also the most probable chlorine atom-pair radial distances and azimuthal angles in various argon pressures.

Traditionally and phenomenologically, the thermalized atompair separations have been evaluated by the approximation relation $m v D_{0} / k T$, in which $v$ is the initial hot atom speed and $D_{0}$ is the mutual diffusion constant, derived from the Langevin equation. ${ }^{51,53}$ Adding the initial bond distance of the chlorine molecule to the above quantities, one could obtain the final chlorine atom separations $\Delta r$. They are also listed in Table 2 . As shown in the table, the most probable radial distances of the MD method are in reasonably good agreement with the atom-pair separations estimated by the Langevin equation, especially in the high-pressure region.

Through photofragment translational spectroscopy,, 22 it is known that at $300 \mathrm{~K}$, due to the molecular rotation, the free chlorine molecules are photodissociated into two chlorine atoms with the maximum angular probability appearing at $8.8^{\circ}$ with respect to their initial molecular axis. Comparing this with the values of $\vartheta_{0(\max )}$ of Table 2, the most probable angle of chlorine atom pair distribution of $0.50 \mathrm{kbar}$ argon pressure is already in agreement with that of molecules dissociated in the free state. Apparently, as the buffer pressure increases, the dissociation of the chlorine molecules are increasingly influenced by argon in the form of the $\mathrm{Ar} / \mathrm{Cl}_{2}$ clusters and/or the argon cage. Phenomenologically, there are no corresponding equations for the description of the azimuth-population relaxation in the photodissociation process.

4B. The Probability Rate Constants of Geminate Recombinations. For chlorine molecules, it has been established that there are four bound states which are correlated to the two ground chlorine atoms: $\mathrm{X}, \mathrm{A}^{\prime}, \mathrm{A}$, and $\mathrm{B}^{\prime}$. All these states have their shares of probability to be populated in the recombination reactions. In the present study, the detailed recombination probability rate constant over the $\mathrm{X}$ state potential shall be considered. The general features of the probability rate 


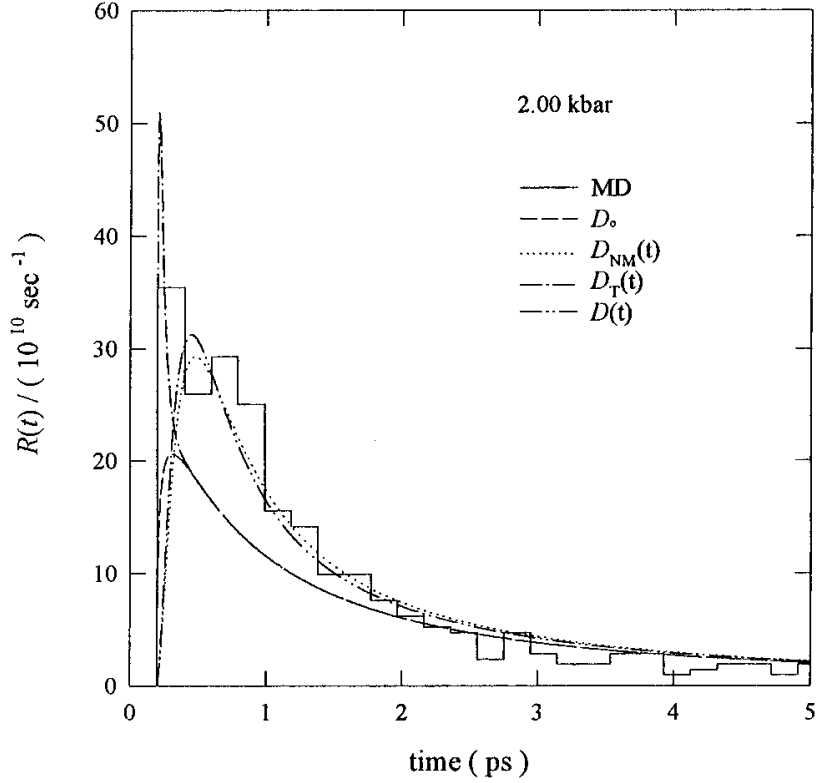

Figure 4. Recombination probability rate constants at 2.00 kbar argon pressure calculated by the MD method, and the diffusion equation in the field-free potential with constant diffusion coefficient $\left(D_{0}\right)$, local transient temperature effect $\left(D_{\mathrm{T}}(t)\right)$, the non-Markovian effect $\left(D_{\mathrm{NM}^{-}}\right.$ $(t)$ ), and the non-Markovian effect approximated by eq 23 . See text for details.

constants for the remaining electronic states are similar to the present calculations, and only their photolytic quantum yields shall be reported in subsection $4 \mathrm{~F}$.

Figures 4 and 5 show the probability rate constants for geminate-pair recombination to the ground $\mathrm{X}$ state at 1.00, 2.00, and $6.00 \mathrm{kbar}$ argon pressures, respectively. The time zero is set at the initiation time of the photodissociation. The events of the MD recombination reactions are recorded when the recombining chorine atom-pair reaches the potential position of $2.5 k T$ below the dissociation energy. In the present case, the distance is $3.58 \AA$. This internuclear distance is also used as the radius of the boundary condition for the diffusion equation. To ensure that the event was a stable recombination process, the trajectories of the chlorine atom pair within the bound state were also taken and checked for at least three additional vibrational periods after they crossed the preset recombination distance. The MD probability rate constants are shown by the histograms in the figures. The corresponding results of the diffusion equation with both the Smoluchowski boundary condition and the initial spatial distribution condition as obtained by the MD method are also shown in the figure. The initial time of the diffusion equation is set at the appearance time of the recombination obtained by the MD method, which is always slightly shorter than the thermalization time defined in the previous subsection. For a comparison between the results of the MD method and the solutions of the diffusion equation, this offset of the initial time for the diffusion equation is needed for counting those recombination events earlier than the mean thermalization time.

As shown in the figures, the diffusion equation with the constant diffusion coefficients consistently yields lower rate constants than the MD values even under the Smoluchowski boundary condition, a condition known to give the upper bound of the more flexible radiation boundary condition.

Several possible factors may account for the lower values of the rate constants obtained by the simplest diffusion equation. Setting aside the possible influences of the parameters in the boundary and initial conditions, recent studies suggest that the
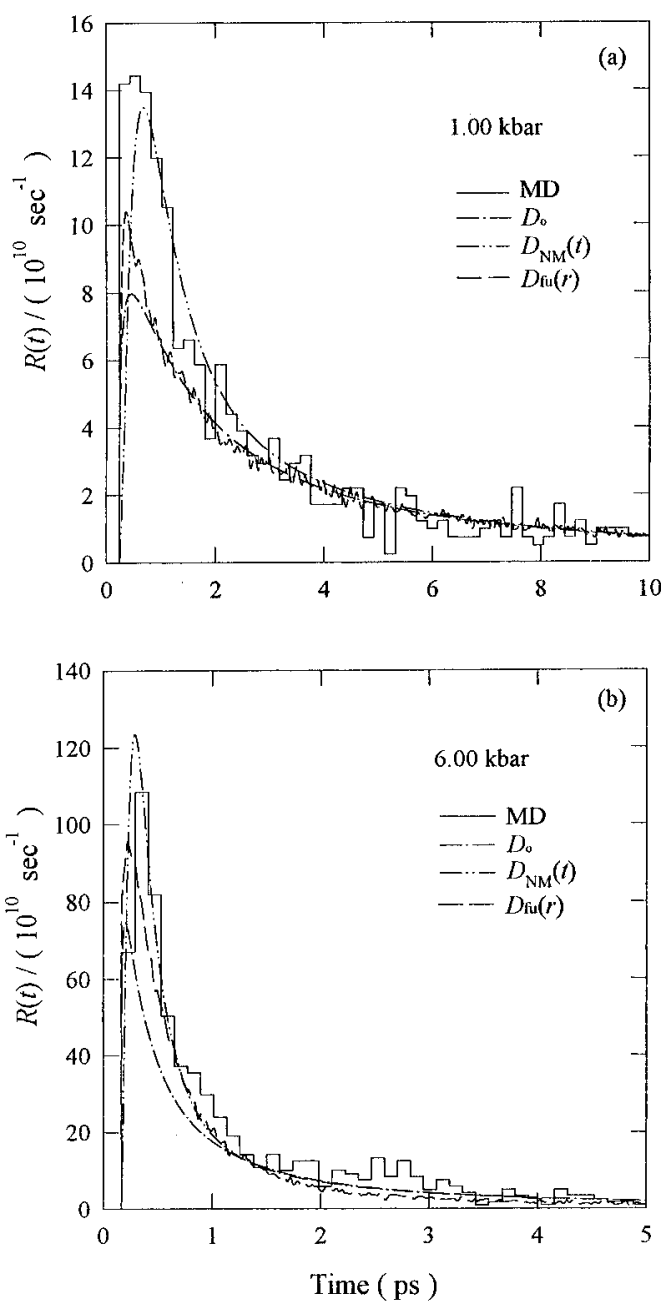

Figure 5. Recombination probability rate constants at 1.00 and 6.00 kbar argon pressure, respectively, calculated by the MD method and the Smoluchowski equation approach with constant diffusion coefficient $\left(D_{0}\right)$ and the non-Markovian effect $\left(D_{\mathrm{NM}}(t)\right)$ in the field-free potential, and also with hydrodynamic interactions in the full mean-force potential $\left(D_{\mathrm{fu}}(r)\right)$, respectively.

transient local heating effect due to the hot atom thermalization process, the non-Markovian effect on the reactant transport process, the mean-force potential, which includes the long-range reactant-reactant interaction, and hydrodynamic interactions are all important factors deserving consideration. ${ }^{26-29,47,48,54}$ Their contributions to the present recombination reactions are evaluated in the following three subsections.

4C. The Effect of the Initial Local Temperature Jump on the Probability Rate Constants of Geminate Recombinations. The local temperature that is related to the average kinetic energy of the Ar atoms at a certain position can be calculated by the MD method. Figure 6 shows the mean local temperature as a function of both the time and the $\mathrm{Ar}-\mathrm{Cl}$ distance at 2.00 kbar Ar pressure. Here the local temperature is the average temperature at a specified Ar radial distance from the chlorine atom. In the present case, the distance varies from 3.25 to 5.25 $\AA$ with a radial shell width of $0.5 \AA$. Note that the mean distance of the first argon shell from the chlorine atom is $4.3 \AA$ at $2.00 \mathrm{kbar}$. Several features emerge from this spatialtemporal temperature profile: (a) At the mean thermalization time $0.460 \mathrm{ps}$, the local temperatures around the chorine atoms also reach the equilibrium temperature; (b) for those argon atoms beyond the distance of the first argon shell, there is no appreciable local temperature heating over the whole thermalization process; in other words, the expected transient heating 


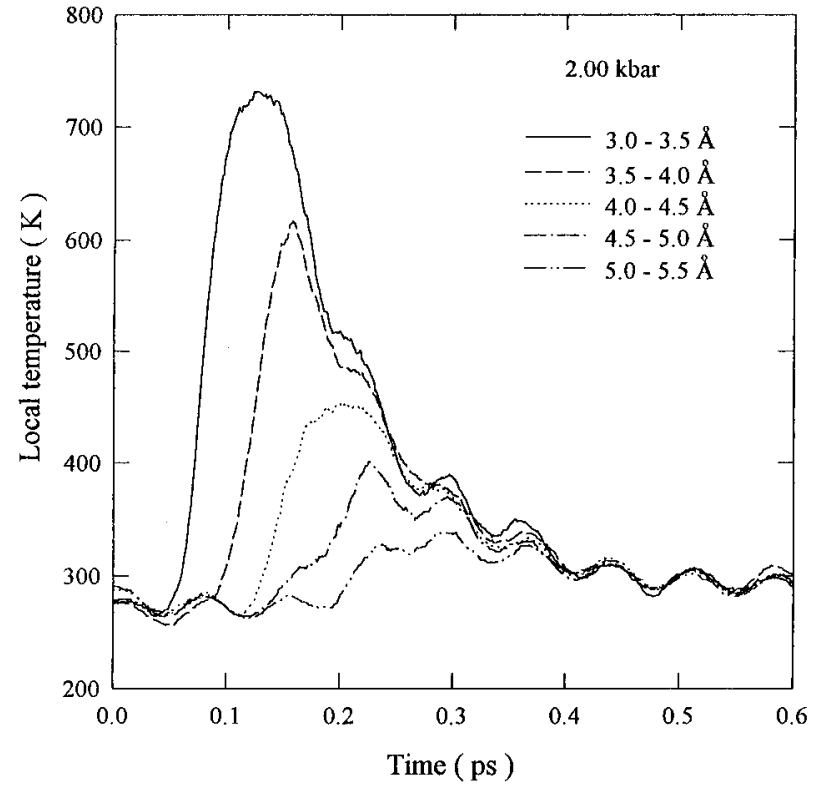

Figure 6. Transient local argon shell temperature as a function of the time and the radial distance from the chlorine atom after the photodissociation at $2.00 \mathrm{kbar}$ argon pressure. The high-frequency modulation of the relaxation is due to the coupling between the system and the thermostat.

phenomenon is localized within the first argon shell; and (c) the local temperatures around the chlorine atom are essentially the same within the mean distance of the first argon shell after the photodissociation of $0.201 \mathrm{ps}$, the time the earliest recombination event occurs in the MD simulations.

Supported by the probability density contour map of Figure 3 , which shows that the majority of the thermalized atom-pairs are within the separation of $8 \AA$, one may conclude that the recombining chlorine atom-pairs as a whole are essentially immersed in a localized heat-bath of a uniform temperature, which decays to the bulk temperature. In other words, in the diffusion equation approach, one may approximate the effect of the local temperature jump on the recombination process simply by incorporating a time-dependent diffusion constant into the diffusion equation instead of the proposed more generalized case, in which the diffusion constant is dependent on both position and time. ${ }^{29}$ For the present purpose, the temperaturedependent diffusion constant could be expressed as a timedependent quantity in the following simple exponential decay form:

$$
D_{\mathrm{T}}(t)=D_{0}+a_{1} \exp \left(-a_{2} t\right)
$$

in which $D_{0}$ is the diffusion constant at $300 \mathrm{~K}$, and $a_{1}$ and $a_{2}$ are parameters. By fitting the equation to the diffusion constants that correspond to the transient temperatures within the first argon shell obtained by the MD calculation, one obtains $a_{1}=$ $3.153 \times 10^{-8} \mathrm{~m}^{2} \mathrm{~s}^{-1}$ and $a_{2}=23.83 \mathrm{ps}^{-1}$. The time zero for the above equation is set at 0.201 ps after the photoexcitation of the chlorine molecules, which is just the earliest appearing recombination time in the MD calculations. The corresponding generalized diffusion equation under the Smoluchowski boundary condition could be solved in closed form. ${ }^{28,51}$ For the $\mathrm{Cl}_{2} /$ Ar case, the probability rate constants and the cumulative rate with the above initial atom-pair distribution become

$$
\begin{aligned}
& R_{\mathrm{s}, \mathrm{T}}(t)=\frac{2 N d \pi^{1 / 2} D_{\mathrm{T}}(t) \bar{D}_{\mathrm{T}}(t)^{-3 / 2}}{c\left(c^{2}+4\right)}(1- \\
& \left.\mathrm{e}^{-c \pi}\right) \int_{R_{0}}^{\infty} \frac{\left(r_{0}-R_{0}\right)^{2}\left(r_{0}-d\right)}{r_{0}} \mathrm{e}^{-\left[\left(r_{0}-d\right)^{2}+4 b r_{0}^{2} D_{\mathrm{T}}(t)\right] / 4 \bar{D}_{\mathrm{T}}(t)} \mathrm{d} r_{0}
\end{aligned}
$$

and

$$
\begin{array}{r}
W_{\mathrm{s}, \mathrm{T}}(t)=\frac{4 \pi N d\left(1-\mathrm{e}^{-c \pi}\right)}{c\left(c^{2}+4\right)} \int_{R_{0}}^{\infty} \frac{\left(r_{0}-R_{0}\right)^{2}}{r_{0}} \mathrm{e}^{-b r_{0}{ }^{2} \times} \\
\operatorname{erfc}\left(\frac{r_{0}-d}{\sqrt{4 \bar{D}_{\mathrm{T}}(t)}}\right) \mathrm{d} r_{0}
\end{array}
$$

with $\bar{D}_{\mathrm{T}}(t)=\int_{0}^{t} \mathrm{dt}^{\prime} D_{\mathrm{T}}\left(t^{\prime}\right)$.

The long-short dashed line of Figure 4 shows the probability rate constant of eq 20 at $2.00 \mathrm{kbar}$ argon pressure. As expected, the increasing of the diffusion constant due to the transient local temperature heating enhances the rate constant at the earliest stage of the recombination reaction, as suggested by the differences between results of the MD and the diffusion equation approaches. This effect is more pronounced for the lower pressure cases, as shown in the figures of 2.00 and $1.00 \mathrm{kbar}$ Ar pressures. However, as shown in Figure 5b, there are no noticeable effects due to the local heating for the $6.00 \mathrm{kbar}$ case. In short, the local temperature jump enhances the earliest recombination rate constant, especially for the lower argon pressure systems. Nevertheless, this effect is not the major factor for the overall mismatch between the MD results and those of the diffusion equation as suggested in Figures 4 and 5 .

4D. The Effect of the Non-Markovian Process on the Probability Rate Constants of Geminate Recombinations. Another factor that could underestimate the short time rate constants is the non-Markovian effect. Dong and Andre formulated this effect in the form of the generalized diffusion equation with a time-dependent diffusion constant. ${ }^{26,28}$ Following the general approach, the final functional forms of the recombination probability rate constants and the cumulative reaction probabilities under the Smoluchowski boundary condition are exactly the same as eqs 20 and 21 obtained for the calculations of the local thermal effect, except that their physical origins of the time-dependent diffusion constants are different. In this study, the analytic time-dependent diffusion constants due to the non-Markovian effect are evaluated in the following manner. To begin with, the time-dependent diffusion constants due to the non-Markovian effect could be formally calculated by the relation ${ }^{28}$

$$
D(t)=\frac{1 \partial}{6 \partial t}\left\langle|\mathbf{r}(t)-\mathbf{r}(0)|^{2}\right\rangle
$$

in which the quantity in brackets is the mean square displacement of a diffusing particle. It is well-known that the functional form of the mean square displacement of a Brownian particle derived from the Langevin equation is ${ }^{51}$

$$
\begin{aligned}
\left\langle|\mathbf{r}(t)-\mathbf{r}(0)|^{2}\right\rangle=\frac{u_{0}^{2}}{\xi^{2}}\left(1-\mathrm{e}^{-\xi t}\right)^{2}+ & \\
& \frac{3 k T}{m \zeta^{2}}\left(2 \zeta t-3+4 \mathrm{e}^{-\zeta t}-\mathrm{e}^{-2 \zeta t}\right)
\end{aligned}
$$

in which $m$ is the particle mass, $m u_{0}^{2} / 2$ is the thermal energy, $m \zeta$ is the friction constant, and, according to the Einstein relation, $D_{0}=k T / m \zeta$. Starting from eq 23, two calculation procedures were employed to evaluate $D(t)$ due to the non- 


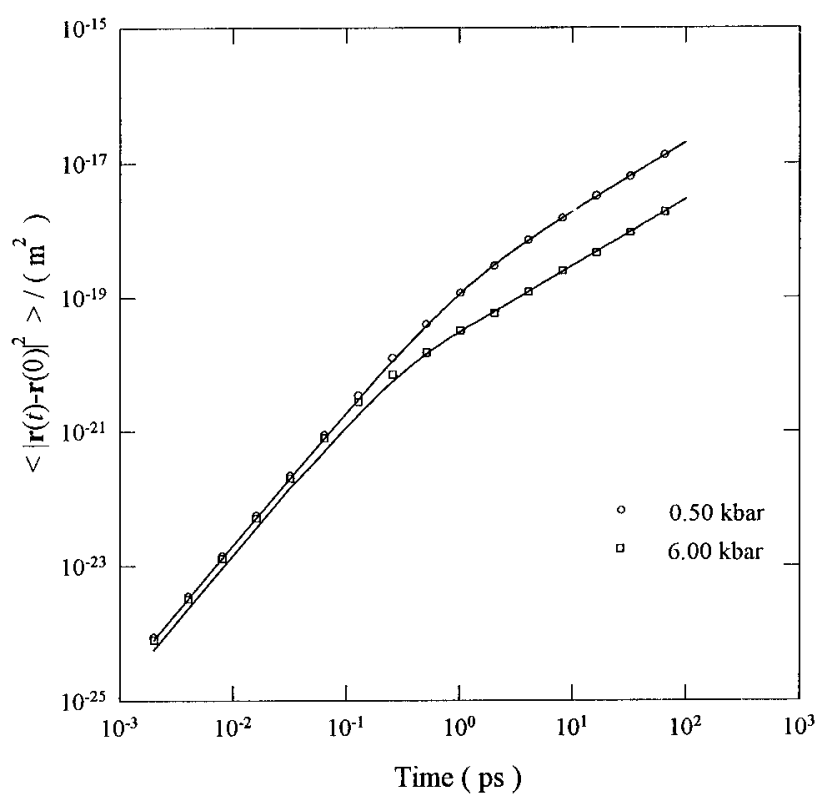

Figure 7. Mean square displacement of the chlorine atom in 0.50 and $6.00 \mathrm{kbar}$ argon calculated by the MD method. The solid lines are the fitted curves of eq 23.

Markovian effect in the $\mathrm{Cl}_{2} / \mathrm{Ar}$ system. One is a direct adapting of the equation with its original interpretations, in which $u_{0}^{2}$ is determined by the average thermal energy and $\zeta$ is calculated by the Einstein relation and $D_{0}$. The corresponding rate constant and cumulative rate could be calculated according to eqs 20 and 21. The result with 2.00 kbar argon pressure is shown as a dashed line indicated as $D(t)$ in Figure 4.

The second approach is based on the time-dependent mean square displacements of the chlorine atom in argon calculated by the MD method. Firstly, $u_{0}^{2}$ was determined by the mean square displacements of the short time regime; secondly, the quantity $6 \mathrm{kT} / \mathrm{m} \zeta$ of eq 23 , which corresponds to the asymptotic diffusion constant, was obtained from the data of the long time regime; and finally, with the fixed $u_{0}^{2}$ and $6 k T / m \zeta$ values, $\zeta$ is independently obtained by fitting eq 23 over the entire range of the mean square displacements. Figure 7 shows the fitted curves at 0.50 and $6.00 \mathrm{kbar}$ argon pressures, respectively. As suggested by the figure, for the present system, the MD results could be adequately represented by the functional form of eq 23. The corresponding time-dependent diffusion constant and then the probability rate constants and cumulative reaction probabilities could be calculated accordingly. Note that in the above calculations of the recombination rate constants, the chlorine mutual diffusion constant, which is just twice the value of the corresponding chlorine-argon diffusion constant, should be used. The probability rate constants are shown in Figures 4 and 5 by the short-dashed line indicated by $D_{\mathrm{NM}}(t)$.

Figures 4 and 5 suggest that although both the transient local temperature jump and the non-Markovian process enhance the probability rate constant of the short time region, the former factor occurs in a much faster time domain, and its importance is less as the pressure increases. The non-Markovian effect is an important factor for a quantitative description of the recombination reactions by the diffusion equations. For practical purposes, at least in the present system, the major contribution of the non-Markovian process could be evaluated in the framework of the generalized diffusion equation with the simple time-dependent diffusion constant directly derived from the Langevin equation.

4E. The Effect of the Mean-Force Potential, the $\mathrm{Cl}-\mathrm{Cl}$ Long-Range Potential, and Hydrodynamic Interactions on the Probability Rate Constants of Geminate Recombinations. Since the interaction potential between $\mathrm{Ar}-\mathrm{Cl}$ is approximated by that of $\mathrm{Ar}-\mathrm{Ar}$ in the present study, the mean-force potential of $\mathrm{Cl}-\mathrm{Cl}$ in the argon buffer gas was calculated at two levels: the mean-force potential due to $\mathrm{Ar}$ and the full mean-force potential that includes the $\mathrm{Cl}-\mathrm{Cl}$ long-range potential. The former potential was calculated directly from the equilibrium distribution function $g(r)$ of the Ar system through

$$
g(r)=\mathrm{e}^{-\beta U(r)}
$$

and is equivalent to the mean-force potential in which the $\mathrm{Cl}-$ $\mathrm{Cl}$ interaction potential is taken to be the same as the $\mathrm{Ar}-\mathrm{Ar}$ van der Waals potential. Adding the real $\mathrm{Cl}-\mathrm{Cl}$ long-range potential to the above mean-force potential and then subtracting the extra van der Waals potential, one obtains the full meanforce potential for the present study.

The hydrodynamic interaction was approximated by the spatially dependent diffusion coefficient ${ }^{47,48}$

$$
D(r)=D_{0}\left(1-\frac{1}{2} \exp \left\{-\frac{r-\sigma}{\sigma}\right\}\right)
$$

in which $\sigma$ is approximated by $3.41 \AA$, the Lennard-Jones radial parameter of the chlorine atom and argon atom, and $D_{0}$ is the asymptotic bulk mutual diffusion coefficient.

With these potentials and diffusion coefficients, the corresponding Smoluchowski equations were then solved by the Brownian dynamics trajectory simulations under the Smoluchowski boundary condition. In the present study, 150000 trajectories were calculated for 2.00-6.00 kbar Ar pressures and 300000 trajectories for $1.00 \mathrm{kbar}$ Ar pressure. Figure 8a shows the probability rate constants of the chlorine atom geminate recombination with either the mean-force potential of $\mathrm{Ar}$ (denoted by the curve of $D_{\mathrm{m}}^{\circ}$ ) or the full mean-force potential that includes the $\mathrm{Cl}-\mathrm{Cl}$ long-range potential (denoted by the curve of $D_{\mathrm{fu}}^{\circ}$ ) under the constant diffusion coefficient $D_{0}$ at $2.00 \mathrm{kbar}$ Ar. The results of the MD and field-free solutions are also included in the figure for reference. The curves of $D_{\mathrm{m}}(r)$ and $D_{\mathrm{fu}}(r)$ shown in Figure $8 \mathrm{~b}$ are the corresponding results with hydrodynamic interactions being approximated by eq 25 . The numerical solutions were presented in their original forms without any smoothing procedure. Several features emerge from the figure. (1) For times shorter than about $1 \mathrm{ps}$, the reaction rate constants of both mean-force potentials are all larger than that of the free-field case; the rate constants of the full mean-force potential are always larger than those without the $\mathrm{Cl}-\mathrm{Cl}$ long-range potential; and the rate constants with hydrodynamic interactions are all smaller than the corresponding rate constants without the interactions. (2) For times longer than about $1 \mathrm{ps}$, a reverse of the above trend is observed. For instance, the rate constants of both mean-force potentials are all smaller than that of the free-field case. (3) The rate constant of the full mean-force potential with hydrodynamic interactions is close to that of the simple mean-force potential with the constant diffusion coefficient, i.e. close to the case without considering both the $\mathrm{Cl}-\mathrm{Cl}$ long-range potential and hydrodynamic interactions. In other words, for the present case, in the short time region, the increasing of the rate constant due to the $\mathrm{Cl}-\mathrm{Cl}$ long-range force is nearly compensated by the decreasing of the rate constant by hydrodynamic repulsion. (4) As shown by Figure $8 b$, the rate constant of the full meanforce potential with hydrodynamic interactions is in good agreement with the MD results in the initial maximum position. The values of the longer time regime are all consistently lower than those of the MD results. 

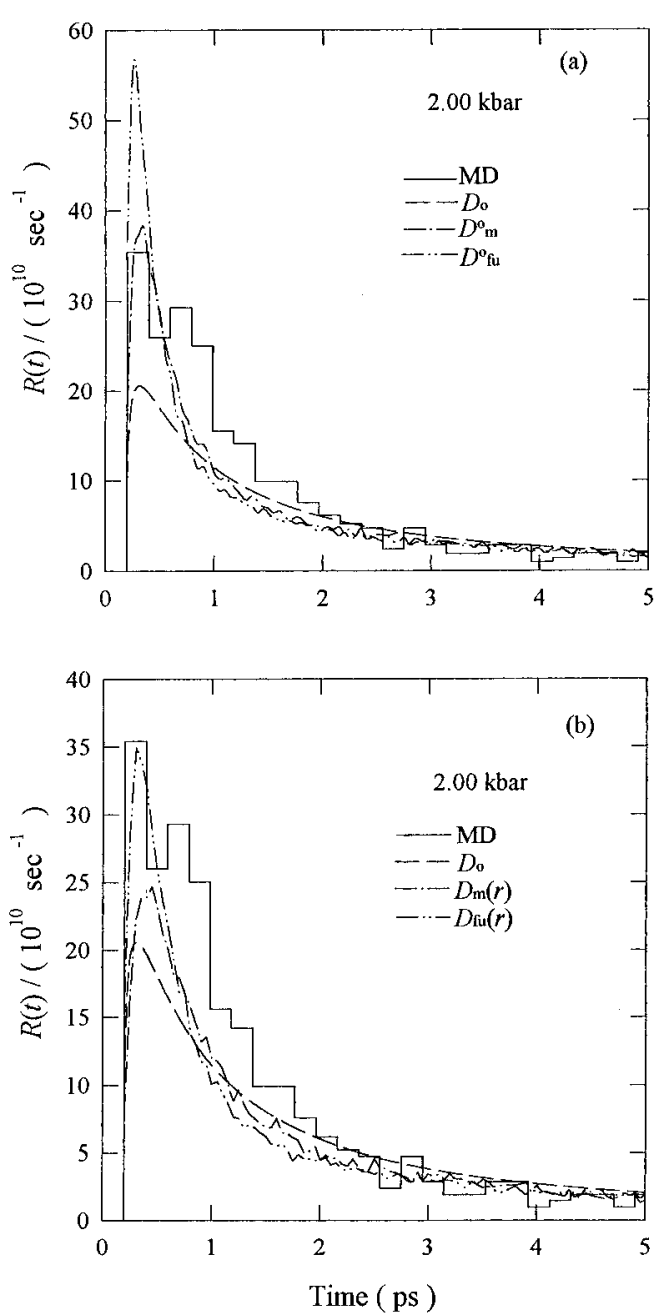

Figure 8. Recombination probability rate constants with constant diffusion coefficient in the mean-force potential $\left(D_{\mathrm{m}}^{\circ}\right)$ and the full meanforce potential including the $\mathrm{Cl}-\mathrm{Cl}$ long-range force $\left(D^{\circ}{ }_{\text {fu }}\right)$, respectively (a) and with hydrodynamic interactions in the corresponding potentials $\left(D_{\mathrm{m}}(r)\right.$ and $\left.D_{\mathrm{fu}}(r)\right)(\mathrm{b})$ at $2.00 \mathrm{kbar}$ Ar. The MD and field-free results (MD and $D_{0}$ ) serve as references.

Comparing the present result of the full mean-force potential with hydrodynamic interactions shown in Figure $8 \mathrm{~b}$ with that of the non-Markovian process shown in Figure 4, one finds that the contributions of these two different types of factors actually show up in two different time domains if one uses the $D_{0}$ curve of the field-free case as a reference: the full mean-force potential with the distance-dependent diffusion constant has a pronounced positive contribution around 0.35 ps and a non-negligible negative contribution in a time range from 1 to $5 \mathrm{ps}$; while the contribution of the non-Markovian effect is all positive, which starts from 0.4 ps and lasts up to about 3 ps. Figure 4 shows that the non-Markovian correction underestimates the initial maximum rate constant while, in the meantime, overestimates the rate constant as the time longer than around 2 ps as compared with the MD results. It appears that these finer mismatches could be accounted for by the contribution of the full meanforce potential with hydrodynamic interactions. Figure 5 also shows the corresponding results at 1.00 and $6.00 \mathrm{kbar} \mathrm{Ar}$ pressures. Their general features due to the non-Markovian effect and the effect of the full mean-force potential with hydrodynamic interactions are similar to those of $2.00 \mathrm{kbar} \mathrm{Ar}$ case. The present study suggests that in the Smoluchowski equation approach for the recombination reactions, the nonMarkovian effect, the mean-force potential, and hydrodynamic
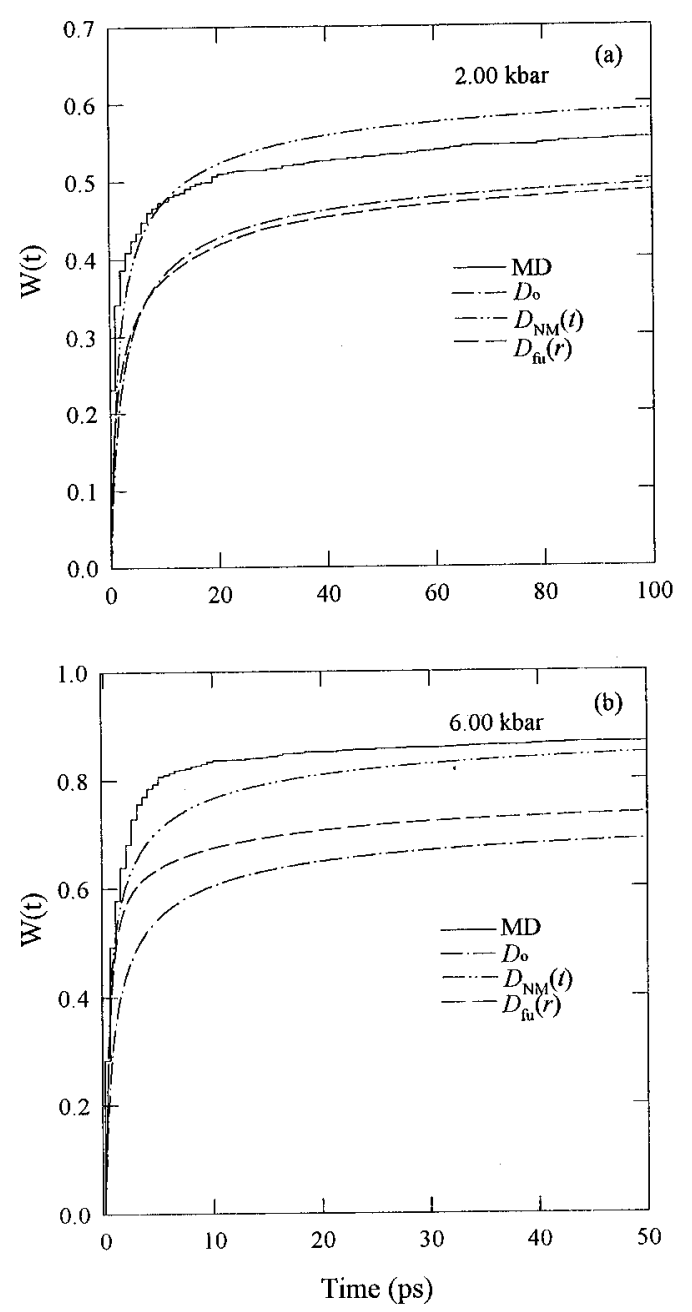

Figure 9. Cumulative recombination probabilities as calculated by the MD method and the Smoluchowski equation approaches with constant diffusion coefficient $\left(D_{0}\right)$ and the non-Markovian effect $\left(D_{\mathrm{NM}}(t)\right)$ in the field-free potential, and also with the hydrodynamic interaction in the full mean-force potential $\left(D_{\mathrm{fu}}(r)\right)$, at 2.00 and $6.00 \mathrm{kbar}$ argon pressures, respectively.

interactions are all important factors and need to be treated in a consistent and unified manner.

4F. The Cumulative Reaction Probabilities and Recombination Quantum Yields of Geminate Recombinations. Figure 9 shows the cumulative reaction probabilities of the recombination reaction in the ground $\mathrm{X}$ state calculated by the $\mathrm{MD}$ and Smoluchowski equation methods, respectively. The Smoluchowski boundary condition was imposed in the diffusion equation approach. As shown in Figure 9, the cumulative recombination probabilities with constant diffusion coefficients are consistently lower than the results of the MD method, a necessary consequence of the comparatively lower values of the probability rate constants discussed in the previous subsections. The incorporation of the non-Markovian contribution remedies the major discrepancy. As expected from the rate constants, for the $2.00 \mathrm{kbar}$ Ar case, the cumulative probability calculated at full mean-force potential with hydrodynamic interactions is initially higher, but later lower than that of the field-free solution. This could account for the finer discrepancy between the solution of the non-Markovian effect and the MD results, as shown in Figure 9a. On the contrary, at 6.00 kbar, the values of the full mean-force potential with hydrodynamic interactions are all higher than those of the field-free solution, which also could account for the deficiency of the nonMarkovian results, as shown in Figure 9b. As indicated in the 
TABLE 3: Recombination Quantum Yields of the Four Bound Electronic States and the Adiabatic and Statistical Quantum Yields of $\mathbf{C l}_{2}$ under $355 \mathrm{~nm}$ Radiation in Argon at $300 \mathrm{~K}$

\begin{tabular}{cllllll}
\hline pressure $^{a}$ & \multicolumn{1}{c}{$\mathrm{X}$} & \multicolumn{1}{c}{$\mathrm{A}^{\prime}$} & \multicolumn{1}{c}{$\mathrm{A}$} & \multicolumn{1}{c}{$\mathrm{B}^{\prime}$} & \multicolumn{1}{c}{$\Phi_{\mathrm{a}}$} & \multicolumn{1}{c}{$\Phi_{\mathrm{s}}$} \\
\hline 0.18 & 0.039 & 0.023 & 0.019 & 0.014 & 0.0086 & 0.039 \\
0.50 & 0.19 & 0.17 & 0.17 & 0.14 & 0.063 & 0.19 \\
1.00 & 0.39 & 0.39 & 0.37 & 0.34 & 0.14 & 0.39 \\
2.00 & 0.55 & 0.64 & 0.57 & 0.53 & 0.22 & 0.55 \\
4.00 & 0.76 & 0.74 & 0.75 & 0.74 & 0.28 & 0.76 \\
6.00 & 0.88 & 0.80 & 0.83 & 0.84 & 0.31 & 0.88
\end{tabular}

${ }^{a}$ Pressure in kbar.

above recombination rate constants, the cumulative recombination probabilities also suggest the necessity of considering the above rate-constant influencing factors in a unified manner. For pressures less than $1.00 \mathrm{kbar}$, both the probability rate constants and recombination quantum yields under the Smoluchowski boundary condition are all larger than the MD results. Apparently, the radiation boundary condition instead of the Smoluchowski one is more appropriate for the recombination reactions at the lower pressure region.

In the MD calculations, the geminate recombination reactions in each bound electronic excited state $-\mathrm{B}^{\prime}, \mathrm{A}, \mathrm{A}^{\prime}$, and $\mathrm{X}-$ are assumed to be following its own adiabatic potential curve. The recombination quantum yields were then calculated for each of the four bound states over the argon pressure range 0.18-6.00 kbar. Table 3 shows that the recombination quantum yields are essentially the same among the four bound electronic states for argon pressure higher than $0.50 \mathrm{kbar}$. However, for the lower pressure region, the recombination quantum yields are dependent on the electronic states: the lower the overall potential well, the higher the recombination quantum yields, if the electronic degeneracies are not counted.

To gain some insight into the recombination reactions in the low-pressure regime, theoretically, one may employ the energy transfer model as proposed by Smith to calculate the relative recombination efficiencies for the low-pressure gas systems. ${ }^{55}$ The absolute rate constants calculated by the model are dependent on the collision efficiencies of the third body during the recombination, whose values are not exactly known. Nevertheless, according to the model, the relative recombination efficiencies of the electronic states are insensitive to this factor and also to the details of the short-range interaction potentials. The comparatively simple method could yield a quantitative account of the relative recombination efficiencies among the bound electronic states. With the experimental potentials of these bound states, especially the long-range parts of the potentials, the relative recombination efficiencies were calculated to be $30 \%, 30 \%, 28 \%$, and $12 \%$ for the $\mathrm{X}, \mathrm{A}^{\prime}, \mathrm{A}$, and $\mathrm{B}^{\prime}$ states, respectively, if the electronic degeneracies are not counted. This means that only the effects of the potentials are considered for comparisons here. The corresponding MD results at 180 bar Ar pressure are $41 \%, 24 \%, 20 \%$, and $15 \%$. Although the agreement in the magnitudes is only fair, both methods yield the same decreasing trend for the recombination efficiencies as the state moves from $\mathrm{X}$ to $\mathrm{B}^{\prime}$. Apparently, the lower the overall electronic potential gets, the more rovibrational states the potential could support near the dissociation energy, and therefore, the larger the recombination quantum yield becomes.

To date, there is only one reported experiment on the measurement of the geminate recombination quantum yields of the chlorine molecules in 25.5-179.3 bar argon pressure at 300 K. ${ }^{13}$ The recombination quantum yield at 179.3 bar was measured to be 0.0050 . Since the nonadiabatic factors among the $\mathrm{Cl}_{2}$ electronic states in the argon gas are still not tractable, to calculate the net recombination quantum yields of $\mathrm{Cl}_{2}$ from the present MD results, two limiting cases-the adiabatic and the statistical quantum yields - are considered. The adiabatic recombination quantum yield is calculated under the assumption that, after photodissociation, the recombination process follows the specific bound adiabatic potential with a population weight proportional to the electronic degeneracy. Since there are 16 electronic states correlated with the two ground chlorine atoms if the degeneracies are also counted, the adiabatic recombination quantum yield of $\mathrm{Cl}_{2} / \mathrm{Ar}$ is defined as

$$
\Phi_{\mathrm{a}}=\frac{1}{16} R_{\mathrm{X}}+\frac{2}{16} R_{\mathrm{A}}+\frac{2}{16} R_{\mathrm{A}^{\prime}}+\frac{1}{16} R_{\mathrm{B}^{\prime}}
$$

in which the $R$ 's are the recombination quantum yields for each specified electronic state. At the other extreme, for high enough argon pressure, the majority of all the recombination events, which take place somewhere in the neighborhood of the near dissociation region, may undergo multiple nonadiabatic transitions to populate the lowest electronic state. The statistical recombination quantum yields are therefore simply defined as $\Phi_{\mathrm{s}}=R_{\mathrm{X}}$ for the present system.

As shown in Table 3, the adiabatic quantum yield at 180 bar is 0.0086 . However, the statistical quantum yield predicts a much larger value, 0.039. Comparing these two values with the experimental result, $0.0050,{ }^{13}$ one could conclude that the adiabatic quantum yield is a better description for the recombination quantum yields at this lower pressure region. Nevertheless, at the high-pressure region, it is expected that the statistical quantum yield would dominate the recombination quantum yields. For instance, the $\mathrm{Br}_{2}$ recombination quantum yields were measured to be 0.80 and 0.81 under 4.05 and 6.10 kbar argon pressure, respectively, at 293 K. ${ }^{6}$ These values are close to the present statistical recombination quantum yields of $\mathrm{Cl}_{2}, 0.76$ and 0.88 under 4.00 and 6.00 kbar, respectively. On the contrary, as shown in Table 3 , the highest adiabatic quantum yield calculated is 0.31 at $6.00 \mathrm{kbar}$, a value too low to account for the experimental observations. If the $\mathrm{Br}_{2}$ measurements could serve as a clue, the switching from the adiabatic to the statistical quantum yields for the chlorine molecules would occur in the argon pressure range $0.3-0.5 \mathrm{kbar}$. Note that there is still a difference between the $\mathrm{Br}_{2}$ and $\mathrm{Cl}_{2}$ systems considered. For $\mathrm{Br}_{2}$, both the bound and repulsive states are involved in the photodissociation process, ${ }^{6}$ while in the present $\mathrm{Cl}_{2}$ case, only one pure repulsive state takes part in the photodissociation process. ${ }^{13}$ In the latter case, recombination reactions could occur only through nonadiabatic transitions among the electronic states involved.

\section{Conclusions}

The photodissociation/recombination reactions of $\mathrm{Cl}_{2} / \mathrm{Ar}$ under $355 \mathrm{~nm}$ radiation at $300 \mathrm{~K}$ are studied by the MD and Smoluchowski equation methods. The thermalization rate constants, chlorine atom-pair spatial distributions, recombination probability rate constants, and recombination quantum yields calculated by the MD methods over the experimental potentials serve as references for quantitative comparisons with the experimental measurements and also the results of the Smoluchowski equation approach. Along this line, the following conclusions can be drawn.

(a) Up to $6.00 \mathrm{kbar} \mathrm{Ar}$ pressure, the chlorine atom-pair separations and the chlorine atom thermalization rate constants predicted by the Langevin equation for the chlorine molecules under $355 \mathrm{~nm}$ radiation at $300 \mathrm{~K}$ are generally a good estimation as compared with the MD results. 
(b) In the photodissociation process, the azimuthal- or $\theta$-dependent distributions of the thermalized chorine atom-pairs in argon, which are originated from the thermal rotational motion of the parent chlorine molecules, still preserve the main angular feature of the chlorine atom-pairs under the free space condition. Although, due to the isotropic nature of the boundary condition, the anisotropic angular distributions do not affect the recombination rate constants treated by the diffusion equation approaches in the present study, one has to take the angular distributions into account if the boundary condition should become latitude-dependent.

(c) The effect of the transient local temperature jump on the recombination probability rate constants, which is due to the hot atom-pair thermalization, was evaluated by the diffusion equation with time-dependent diffusion constants. It increases the recombination rate constants in the initial recombination time region. However, this effect is not significant for the overall recombination process, especially in the high-pressure region.

(d) The non-Markovian effect, which was evaluated in the framework of the generalized diffusion equation, is found to be important for a quantitative analysis of the recombination probability rate constants and quantum yields of the $\mathrm{Cl}_{2} / \mathrm{Ar}$ system over the medium to high-pressure range. However, this approach still underestimates the recombination rate constants at the initial time stage and, meanwhile, overestimates the rate constants in the medium time domain for the medium-pressure systems and, except for the initial transient period, underestimates the rate constants over the medium to long time domain in the high-pressure cases.

(e) The effects of the mean-force potential, the $\mathrm{Cl}-\mathrm{Cl}$ longrange potential, and hydrodynamic interactions, which were evaluated by solving the corresponding Smoluchowski equation, could account for the deficiency left by the results of the nonMarkovian effect. It is suggested that, for a quantitative description of the recombination reactions, a unified treatment of these factors is needed in the diffusion equation approach.

(f) The agreement between the measured quantum yield and the adiabatic quantum yield of the MD calculations at $180 \mathrm{bar}$ argon pressure suggests that the geminate recombination generally follows the adiabatic bound potentials in the low-pressure regime. For the high-pressure regime, with the help of the nonadiabatic transitions among the electronic states in the far internuclear distance region, recombination along the lowest electronic potential is suggested for the $\mathrm{Cl}_{2} / \mathrm{Ar}$ system.

Acknowledgment. The financial support of this work by the National Science Council, Republic of China, is gratefully acknowledged.

\section{References and Notes}

(1) Hippler, H.; Luther, K.; Troe, J. Chem. Phys. Lett. 1972, 16, 174. (2) Hippler, H.; Luther, K.; Troe, J. Ber. Bunsen-Ges. Phys. Chem. 1973, 77, 1104.

(3) Luther, K.; Troe, J. Chem. Phys. Lett. 1974, 24, 85.

(4) Hippler, H.; Troe, J. Int. J. Chem. Kinet. 1976, 8, 501.
(5) Dutoit, J.-C.; Zellweger, J.-M.; van den Beger, H. J. Chem. Phys. 1983, 78,1825 .

(6) Hippler, H.; Schubert, V.; Troe, J. J. Chem. Phys. 1984, 81, 3931.

(7) Paige, M. E.; Russell, D. J.; Harris, C. B. J. Chem. Phys. 1986, 85, 3699.

(8) Paige, M. E.; Harris, C. B. Chem. Phys. 1990, 149, 37.

(9) Lienau, Ch.; Williamson, J. C.; Zewail, A. H. Chem. Phys. Lett. 1993, 213, 289.

(10) Lienau, Ch.; Zewail, A. H. Chem. Phys. Lett. 1994, 222, 224.

(11) Lienau, Ch.; Zewail, A. H. J. Chim. Phys. 1995, 92, 566.

(12) Chang, L.-C.; Song, T.-T.; Tai, C.-C.; Su, T.-M. J. Phys. Chem. 1996, 100, 13548.

(13) Song, T.-T.; Su, T.-M. J. Phys. Chem. 1996, 100, 13554.

(14) Amar, F. G.; Berne, B. J. J. Phys. Chem. 1984, 88, 6720.

(15) Li, Z.; Borrmann, A.; Martens, C. C. J. Chem. Phys. 1992, 97, 7234.

(16) Liu, Q.; Wang, J.-K.; Zewail, A. H. J. Phys. Chem. 1995, 99, 11321

(17) Li, Z.; Zadoyan, R.; Apkarian, V. A.; Martens, C. C. J. Phys. Chem. 1995, 99, 7453 .

(18) Zadoyan, R.; Li, Z.; Martens, C. C.; Apkarian, V. A. J. Chem. Phys.

1994, 101, 6648.

(19) Wang, J.-K.; Liu, Q.; Zewail, A. H. J. Phys. Chem. 1995, 99, 11309.

(20) Nose, S, J. Chem. Phys. 1984, 81, 511.

(21) Nose, S. Mol. Phys. 1984, 52, 255.

(22) Hoover, W. G. Phys. Rev. 1985, A31, 1695.

(23) Otto, B.; Schroeder, J.; Troe, J. J. Chem. Phys. 1984, 81, 202.

(24) Noyes, R. M. Z. Electrochem. 1960, 64, 153.

(25) Noyes, R. M. Progr. React. Kinet. 1961, 1, 131

(26) Dong, W.; Baros, F.; Andre, J. C. J. Chem. Phys. 1989, 91, 4643.

(27) Zhou, H.-X.; Szabo, A. J. Chem. Phys. 1991, 95, 5948.

(28) Dong, W.; Andre, J. C. J. Chem. Phys. 1994, 101, 299.

(29) Strehlow, H. Ber. Bunsen-Ges. Phys. Chem. 1993, 97, 189.

(30) Allen, M. P.; Tildesley, D. J. Computer Simulation of Liquids; Clarendon Press: Oxford, 1987.

(31) Becker, C. H.; Valentini, J. J.; Casavecchia, P.; Sibener, S. J.; Lee, Y. T. Chem. Phys. Lett. 1979, 61, 1.

(32) Casavecchia, P.; He, G.; Sparks, R. K.; Lee, Y. T. J. Chem. Phys. 1981, 75,710 .

(33) Hwang, C.-J.; Jiang, R.-C.; Su, T.-M. J. Chem. Phys. 1986, 84, 5095.

(34) Evard, D. D.; Cline, J. I.; Janda, K. C. J. Chem. Phys. 1988, 88, 5433.

(35) Clyne, M. A. A.; Coxon, J. A. J. Mol. Spectrosc. 1970, 33, 381.

(36) Gibson, G. E.; Rice, O. K.; Bayliss, N. S. Phys. Rev. 1933, 44, 193.

(37) Douglas, A. E.; Hoy, A. R. Can. J. Phys. 1975, 53, 1965.

(38) Ishiwata, T.; Ishiguro, A.; Obi, K. J. Mol. Spectrosc. 1991, 147, 300 .

(39) Tellinghuisen, P. C.; Guo, B.; Chakraborty, D. K.; Tellinghuisen, J. J. Mol. Spectrosc. 1988, 128, 268.

(40) Ishiwata, T.; Kasai, Y.; Obi, K. J. Chem. Phys. 1991, 95, 60

(41) Vargaftik, N. B. Tables on the thermophysical properties of liquids and gases in normal and dissociated states, 2nd ed.; Halsted Press: New York, 1975.

(42) Michels, A.; Wijker, H.; Wijker, H. Physica 1949, 15, 627.

(43) Robertson, S. L.; Babb, S. E., Jr.; Scott, G. J. J. Chem. Phys. 1969 50, 2160.

(44) Durbin, L.; Kobayashi, R. J. Chem. Phys. 1962, 37, 1643.

(45) Scheider, W. J. Phys. Chem. 1972, 76, 349.

(46) Northrup, S. H.; Hynes, J. T. J. Chem. Phys. 1978, 68, 3203

(47) Northrup, S. H.; Hynes, J. T. J. Chem. Phys. 1979, 71, 871.

(48) Northrup, S. H.; Hynes, J. T. J. Chem. Phys. 1979, 71, 884.

(49) Ermak, D. L.; McCammon, J. A. J. Chem. Phys. 1978, 69, 1352.

(50) Zhou, H.-X. J. Phys. Chem. 1990, 94, 8794.

(51) McQuarrie, D. A. Statistical Mechanics; Harper and Row: New York, 1976

(52) Busch, G. E.; Wilson, K. R. J. Chem. Phys. 1972, 56, 3638.

(53) Monchick, L. J. Chem. Phys. 1956, 24, 381.

(54) Wolynes, P. G.; Deutch, J. M. J. Chem. Phys. 1976, 65, 450.

(55) Smith, I. W. M. Int. J. Chem. Kinet. 1984, 16, 423. 